Bundesgesundheitsbl $2021 \cdot 64: 560-567$ https://doi.org/10.1007/s00103-021-03311-2 Eingegangen: 16. Oktober 2020

Angenommen: 12. März 2021

Online publiziert: 9. April 2021

(c) Der/die Autor(en) 2021

Annalena Bußkamp' • Claudia Vonstein' • Judith Tillmann' • Christin Roßmann' • Freia De Bock ${ }^{2}$

${ }^{1}$ Referat 2-22 „Zusammenarbeit mit Ländern, Krankenkassen und Verbänden, Gremien; Gesundes Alter; Frauengesundheit; Männergesundheit", Bundeszentrale für gesundheitliche Aufklärung, Köln, Deutschland

${ }^{2}$ Abteilung 2, Bundeszentrale für gesundheitliche Aufklärung (BZgA), Köln, Deutschland

\title{
Wissenstranslation am Beispiel Bewegungsförderung von älteren Menschen: Wie gelangen wissenschaftliche Erkenntnisse in die kommunale Praxis?
}

gen, Standardarbeitsweisen und anekdotischen Erzählungen basieren [9-11], die teilweise im Gegensatz zu wissenschaftlichen Prinzipien $[11,12]$ und einer evidenzinformierten Entscheidungsfindung [1] stehen. Die Berücksichtigung von WE in der Praxis hat den Vorteil, dass mit einer höheren Wahrscheinlichkeit wirksame Public-Health-Maßnahmen durchgeführt, Opportunitätskosten gesenkt und folglich öffentliche und private Ressourcen effizienter genutzt werden können [9]. Wissenstranslation ${ }^{2}$ kann hier zu einem besseren Verständnis, einer breiteren Nutzung und Akzeptanz der WE in der Praxis, zur Verringerung der Lücke zwischen Wissen aus der Forschung und Umsetzung in der Praxis und somit zur Verbesserung der Public-Health-Maßnahmen führen [13, 14]. Laut Straus et al. [3] meint Wissenstranszu den WE und deren praxisferne bzw. unzureichend an den Bedürfnissen der Nutzenden orientierte Aufbereitung in Bezug auf Länge, Fachjargon und Inhalte [2, 4-8]. Bisherige Untersuchungen zeigen, dass Entscheidungen in der Praxis insbesondere auf persönlichen Erfahrun-

\footnotetext{
1 Evidenzinformierte Entscheidungsfindung meint den Prozess der Gewinnung und Verbreitung von bestverfügbaren WE und die Nutzung dieser zur Information und Verbesserung der Public-Health-Bemühungen.
}

\footnotetext{
2 Aktivitäten, die darauf abzielen, die Nutzung von WE in die Praxis zu steigern, wurden von anderen je nach Disziplin und Wissenschaftsfeld durch vielfältige Begriffe (z. B. Wissenstransfer, Forschungsimplementierung) beschrieben [14, $16,37]$. Für die Zwecke dieser Studie wird der Begriff "Wissenstranslation“ verwendet und so verstanden, dass er die oben genannten Begriffe umfasst. "Wissen“ inkludiert hier kontextabhängige Informationen und wissenschaftliche Forschung, während der Begriff "Translation" sich auf den Prozess der Übertragung des Wissens bezieht.
}

lation die Anwendung von Wissen in der Praxis und bei der Entscheidungsfindung durch AK. Die Canadian Institutes of Health Research führen diese Definition weiter und bezeichnen sie als jeden dynamischen, iterativen Prozess, der die Synthese, die Disseminierung, den Austausch und die ethisch angemessene Anwendung von Wissen beinhaltet, um die Gesundheit der Bevölkerung zu verbessern, effektivere Gesundheitsservices und -produkte bereitzustellen und das Gesundheitssystem zu stärken [15]. Der Prozess ist dabei in ein komplexes System aus Interaktionen zwischen Wissensnutzenden und Forschenden eingebettet, die in ihrem Ausmaß an Engagement, Intensität und Komplexität in Abhängigkeit der Bedürfnisse, Ergebnisse und Art der Forschung variieren [14-16].

Insbesondere im internationalen Kontext wird über Wissenstranslation und die Aufbereitung von WE publiziert. Diese ersten Erkenntnisse lassen sich jedoch nicht uneingeschränkt auf Deutschland und das Feld der Bewegungsförderung übertragen. Der Grund hierfür sind unter anderem kontextuelle Rahmenbedingungen des Gesundheitssystems, föderale Strukturen, unterschiedliche AK und Schwerpunkte der Public-HealthBemühungen. Die vorliegende Studie untersucht daher unserer Kenntnis nach zum ersten Mal in Deutschland, welche 


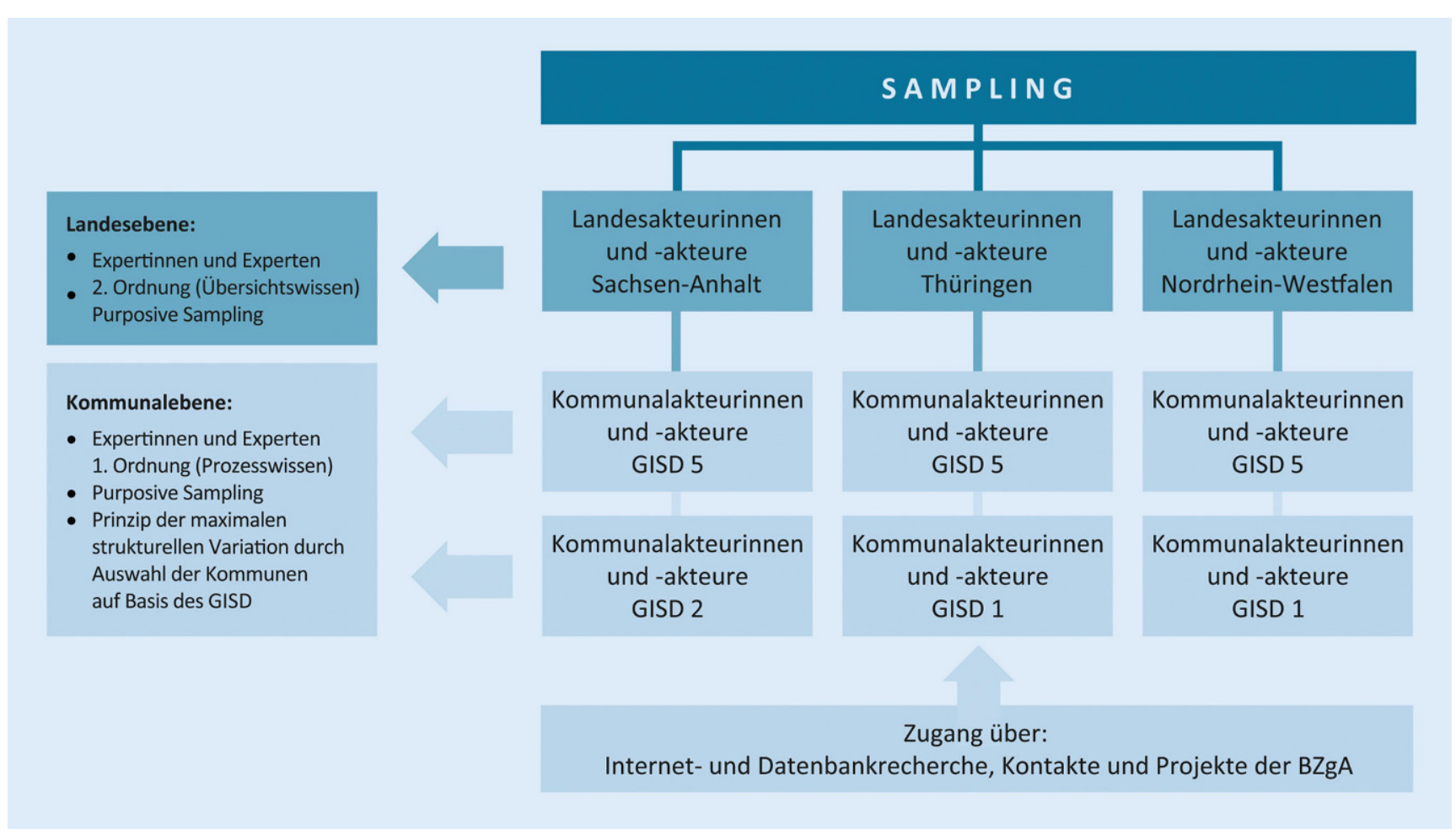

Abb. 1 ॥ Sampling der qualitativen Studie (eigene Darstellung). GISD German Index of Socioeconomic Deprivation; GISD ist der sozioökonomische Deprivationsindex für Deutschland. Die Skala reicht von 1 bis 5 ( 5 = starke sozioökonomische Deprivation, 1 = kaum vorhandene sozioökonomische Deprivation)

konkreten Bedürfnisse zur Aufbereitung und Bereitstellung von WE kommunale AK der Bewegungsförderung haben und wie die Nutzung von WE in der Praxis durch verbesserte Translation erleichtert werden kann. Die AK sind dabei Personen aus der Praxis, die für die Planung, Umsetzung und Strukturentwicklung von Maßnahmen der kommunalen Bewegungsförderung von älteren Menschen in Lebenswelten zuständig sind und zum Teil (politische) Entscheidungskompetenzen besitzen. Im Rahmen des Programms „Älter werden in Balance“ der Bundeszentrale für gesundheitliche Aufklärung (BZgA), welches sich mit der beschriebenen Akteursgruppe beschäftigt (siehe auch https://www.aelterwerden-in-balance.de), sollen hierzu folgende wissenschaftliche Fragestellungen beantwortet werden:

- Wie beschaffen kommunale AK Informationen und Daten zu möglichen Maßnahmen der Bewegungsförderung von älteren Menschen?

- Welchen Nutzen sehen diese in WE?

- Welche Barrieren bestehen bei der Beschaffung und Nutzung von WE?
- In welcher Form müssen WE aufbereitet sein, um praxisrelevant und verständlich für AK der Bewegungsförderung zu sein?

Die vorliegende Studie orientiert sich dabei an einem konzeptionellen Rahmen von Ellen et al. [17], der 7 Elemente zur Schließung der beschriebenen Lücke enthält:

1. Berücksichtigung des lokalen Kontexts,

2. Aufbau von Beziehungen zwischen Forschenden und AK,

3. Erstellung von relevantem und aktuellem Wissen,

4. „Push“-Aktivitäten ${ }^{3}$ von Forschenden,

5. Erleichterung von „Pull“-Bemühungen,

6. „Pull“-Aktivitäten der AK,

7. Evaluation der genannten Aktivitäten.

3 "Push"-Aktivitäten sind Bemühungen von Forschungsorganisationen zur Verbreitung von WE, während „Pull“-Aktivitäten Maßnahmen von AK für den Zugang zu und die Nutzung von WEsind.
Die Arbeit untersucht insbesondere das dritte Element.

\section{Methodisches Vorgehen}

Zur Erforschung des Gebietes wird ausgehend von der Consolidated-Criteria-forReporting-Qualitative-Research-Checkliste [18] auf ein qualitatives Forschungskonzept zurückgegriffen. Dieses enthält als Instrument der Datenerhebung leitfadengestützte Experteninterviews mit offenen Leitfragen und Reflexionsbögen und stützt sich methodologisch auf die qualitative Inhaltanalyse von diesen. Vor den Interviews wurden Kurzfragebögen mit soziodemografischen Daten ausgefüllt, um die Heterogenität der AK abzubilden. Die Expertinnen und Experten sind Mitarbeitende von Kommunalverwaltungen (AK erster Ordnung ${ }^{4}$ [19]) sowie von nichtstaatlichen Landes-

${ }^{4}$ Der wissenssoziologische Expertenbegriff ermöglicht unter Bezugnahme des methodischrelationalen Ansatzes eine Differenzierung in Expertinnen und Experten erster und zweiter Ordnung. Experten und Expertinnen erster Ordnung sind diejenigen, die insbesondere 
Bundesgesundheitsbl 2021 - 64:560-567 https://doi.org/10.1007/s00103-021-03311-2

(c) Der/die Autor(en) 2021

A. Bußkamp · C. Vonstein · J. Tillmann · C. Roßmann · F. De Bock

\section{Wissenstranslation am Beispiel Bewegungsförderung von älteren Menschen: Wie gelangen wissenschaftliche Erkenntnisse in die kommunale Praxis?}

\begin{abstract}
Zusammenfassung
Hintergrund. Wissenschaftliche Ergebnisse können eine Wissensquelle für kommunale Akteurinnen und Akteure der Bewegungsförderung sein, finden jedoch aufgrund von vielfältigen Barrieren selten Anwendung. Wissenstranslation kann diesen Prozess vereinfachen, setzt aber das Erfassen der bisher kaum erforschten Bedürfnisse der Akteurinnen und Akteure voraus.

Ziel der Arbeit. Ziel der qualitativen Studie ist es, die Zugangswege der Akteurinnen und Akteure zu Informationen und wissenschaftlichen Erkenntnissen zu erfassen, mögliche Barrieren zu identifizieren sowie die Bedürfnisse der praktisch Anwendenden bezüglich der Darstellung und Aufbereitung herauszustellen.
\end{abstract}

gen, Filterfunktionen, Herausarbeiten von praxisrelevanten Elementen und Wegen der Bereitstellung.

Diskussion. Für eine erfolgreiche Wissenstranslation sind die Zusammenarbeit und der interaktive Austausch zwischen Wissenschaft, Politik und Praxis sowie die bedarfsgerechte Aufbereitung von wissenschaftlichen Erkenntnissen zentral. Das Vernetzen sowie Bündeln von Wissen auf einer Plattform sind wichtige Aufgaben für die Zukunft.

\section{Schlüsselwörter}

Evidenzbasierte Praxis · Körperliche Aktivität · Gesundheitsförderung · Public Health . Gesundes Altern

\section{Promotion of physical activity among the elderly as an example of knowledge translation: How do scientific findings enter community practice?}

\section{Abstract}

Background. Scientific findings can be an important source of knowledge for public health stakeholders involved in promoting physical activity, but several barriers hinder their use. Knowledge translation can simplify this process, but it requires the understanding of the stakeholder's needs.

Objectives. This qualitative study aims to describe how public health stakeholders access information and scientific findings, identify possible barriers, and highlight the needs of stakeholders in terms of presentation and processing.

Materials and methods. Semi-structured interviews were conducted with twelve local- and state-level stakeholders from North Rhine-Westphalia, Saxony-Anhalt, and Thuringia working in the area of physical activity promotion. The interviewees were selected through purposive sampling. The interviews were evaluated using qualitative content analysis.

Results. The benefits of scientific findings are emphasized by the interviewees, but a lack of resources in combination with a flood of information, high complexity, and technical jargon complicate their application. There is a need for tailored preparation in the form of summaries, filter functions, elaboration of practice-relevant elements, and ways of provision.

Conclusions. To achieve successful knowledge translation, collaboration and interactive exchange between researchers, policymakers, and practice as well as a demand-oriented processing of scientific findings are central. Networking and bundling of knowledge on a platform are important tasks for the future.

\section{Keywords}

Evidence-based practice - Physical activity Health promotion · Public health · Healthy aging verbänden (AK zweiter Ordnung) aus Nordrhein-Westfalen, Sachsen-Anhalt und Thüringen. Sie alle weisen Expertise im Bereich der Bewegungsförderung von älteren Menschen auf.

Die AK auf Landesebene sind stark mit kommunalen $\mathrm{AK}$ vernetzt und ver-

praxeologisches Betriebswissen, also Prozesswissen, besitzen. Expertinnen und Experten zweiter Ordnung sind solche, die abstrakt-reflexives Kontextwissen, ergo Überblickswissen, aufweisen. fügen über ein großes Wissen bzgl. der vorhandenen Strukturen und Probleme. Die kommunalen AK (Verwaltungsmitarbeitende) kennen die präzisen Entscheidungsabläufe in ihren Kommunen, weshalb sie Auskunft über das eigene Handlungsfeld sowie die Bedürfnisse aus der Praxis geben können. Die für diese Studie ausgewählten AK arbeiten in Gesundheitsämtern, Bauämtern, dem Bürgerservice, Fachbereichen für Soziales, Arbeit und Senioren und
Beratungsstellen für Sport- und Bewegungsbetreuung.

Basierend auf vorhandenen WE [2, 7, $8,13,20]$ und eigenen Erfahrungen im Rahmen des Programms „Älter werden in Balance" wurde ein semistrukturierter Interviewleitfaden erstellt. Die Expertinnen und Experten auf Landesebene wurden über bestehende Kontakte und Kooperationen gezielt ausgewählt (Purposive Sampling). Expertinnen und Experten auf Kommunalebene wurden auf Basis der größtmöglichen Variation 
Tab. 1 Beschreibung der zentralen Ergebnisse anhand des Kategoriensystems mit beispielhaften Zitaten

\begin{tabular}{|c|c|c|c|}
\hline $\begin{array}{l}\text { Kate- } \\
\text { gorie }\end{array}$ & $\begin{array}{l}\text { Beschreibung der } \\
\text { Kategorie }\end{array}$ & $\begin{array}{l}\text { Sub- } \\
\text { kategorie }\end{array}$ & $\begin{array}{l}\text { Beschreibung der } \\
\text { Subkategorie }\end{array}$ \\
\hline \multirow[t]{2}{*}{$\begin{array}{l}\text { Informations- } \\
\text { beschaffung }\end{array}$} & $\begin{array}{l}\text { Prozess der Gewin- } \\
\text { nung von Informa- } \\
\text { tionen, d.h. von } \\
\text { kontextabhängi- } \\
\text { gen Daten, der die } \\
\text { folgenden Schritte } \\
\text { enthält: }\end{array}$ & $\begin{array}{l}\text { Rahmenbe- } \\
\text { dingungen }\end{array}$ & $\begin{array}{l}\text { Faktoren, die die Infor- } \\
\text { mationsbeschaffung } \\
\text { der kommunalen Ak- } \\
\text { teurinnen und Akteure } \\
\text { positiv und negativ } \\
\text { beeinflussen }\end{array}$ \\
\hline & $\begin{array}{l}\text { Erschließen von In- } \\
\text { formationsquellen } \\
\text { Sammeln von } \\
\text { Informationen } \\
\text { Aufbereiten von } \\
\text { Informationen } \\
\text { Bewerten von } \\
\text { Informationen }\end{array}$ & Zugang & $\begin{array}{l}\text { Zugangswege und } \\
\text { Wissensträger in Kom- } \\
\text { munen, über welche } \\
\text { sich kommunale Ak- } \\
\text { teurinnen und Ak- } \\
\text { teure Informationen } \\
\text { zur kommunalen Be- } \\
\text { wegungsförderung } \\
\text { beschaffen }\end{array}$ \\
\hline
\end{tabular}

Wissenschaft- Bestverfügbare liche Erkennt- Ergebnisse aus nisse wissenschaftlichen Studien, von Fachinstitutionen oder Fachleuten, die zur Beantwortung einer wissenschaftlichen Fragestellung mithilfe von qualitativen und/oder quantitativen Methoden erhoben wurden
Nutzen Vorteil, der durch die Anwendung von wis senschaftlichen Erkenntnissen im Berufsalltag der kommunalen Akteurinnen und Akteure entsteht

Einbezug Beachtung von wisin Entschei- senschaftlichen Erdungen kenntnissen bei Entscheidungen über verschiedene Handlungsalternativen der kommunalen Bewegungsförderung

Barrieren Faktoren, die die Nutzung und Anwendung von wissenschaftlichen Erkenntnissen in der kommunalen Bewegungsförderung behindern

Strukturelle Für kommunale AkAufbereitung

Inhaltliche Für kommunale AkAufberei- teurinnen und Akteure tung

Bereitstellung schaftlichen Erkenntnisse
Zitat

„Ich ... [realisiere zwar], dass Informationen da sind ..., aber ich habe gar keine Zeit, mich da innerlich mit zu befassen, und habe ... 2 Schrankbereiche, wo ich immer alles hinlege und sage später mal. Und das wird immer später" (Person 1)

„Jemand, der in der Stadtverwaltung arbeitet, kann mit ... [wissenschaftlichen Erkenntnissen], was ganz anderes anfangen, als jemand, der in einer Senioreneinrichtung sich um Nachmittage kümmert" (Person 3)

„Wenn es mal um konkretere Sachen geht, fragt man auch mal Mister Google" (Person 8)

„Grundsätzlich [gibt es] verschiedene Möglichkeiten, über die zuständigen Ministerien und Städte- und Gemeindebund an ... [Informationen] heranzukommen" (Person 5)

„... zumal dann auch die politischen Gremien danach fragen, warum wir was machen. Wenn wir sagen, wir haben hier eine Studie von X, Y, aufgrund dessen bauen wir das auf, dann ist das schon etwas ganz anderes, als wenn wir sagen, ja wir haben uns mal etwas überlegt. Also daher sind solche Studien für uns schon Argumentationshilfe und auch Umsetzungshilfe" (Person 1)

„Mit der wissenschaftlichen Grundlage ... kann man ... Begründungen liefern für die weitere Arbeit ... Sonst heißt es .... Ihr schwimmt wohl im eigenen Saft. Habt ihr euch das selber ausgedacht? Nein, man muss sportliche Entwicklungsmaßnahmen in einer Kommune ... immer auf so einer Basis ... relativ neuer wissenschaftlicher Erkenntnisse [machen]" (Person 5)

„Das kommt auf die [Akteurinnen und] Akteure in den Verwaltungsstrukturen an. Also ich habe gute Erfahrungen bei manchen Gesundheitsämtern“ (Person 3)

„Wenn es darum geht, eine Vorlage auch für den Stadtrat ... vorzubereiten, müssen die [Verwaltungsmitarbeitenden] ja wissen, wovon sie reden, damit das im Stadtrat dann nicht einfach plattgemacht wird" (Person 12)

„Wenn das zu intensiv beschrieben wird ... mit so vielen Fachbegriffen, wo ich fast einen Duden daneben liegen habe, um zu verstehen, was derjenige damit meint. Das hindert mich dann schon so eine Studie von A bis Z zu lesen" (Person 1)

„Ich muss Ihnen ganz ehrlich sagen, ich habe bisher noch ... wirklich keine Barriere erlebt" (Person 2)

„Es muss halt wirklich kurz und knapp [sein], weil alles andere, das merke ich halt selber in meinem Alltag, lese ich dann selten" (Person 10) "Zwischen 20 und 30 Seiten lese ich dann schon mal, wenn der Inhalt umfassend ist, speziell auch ... auf die Region ..., zum Beispiel ländliche Region, zugeschnitten ist" (Person 5)

„... dann einfach [schreiben] ..., was herausgekommen ist ... Weil meistens gibt es ja ein Ergebnis, das lässt sich ... in einem Satz zusammenfassen, auch wenn man das nicht hören möchte" (Person 10)

„Es gibt Tausende gute Beispiele und ich weiß aber auch, dass viele Akteure sofort das Gefühl haben, okay, die und die Ausgangslage ist nicht gleich, das heißt, ich kann damit gar nichts anfangen" (Person 3)

„Am liebsten würde ich mir jemanden wünschen, der an einem Telefon sitzt und der von den Kommunen angerufen werden kann, wo derjenige sagen kann, also spezifisch für meine Kommune bräuchte ich das, das und das" (Person 3)

„Newsletter ist schon okay. Das machen ja inzwischen viele, da haben wir auch Zugang zu und das ist was, da kann man mal schnell ... überfliegen. Ist was Interessantes für mich dabei, dann kann ich es mir rausziehen" (Person 8) 


\begin{tabular}{|c|c|c|}
\hline Variable & Anzahl Teilnehmende $n$ (\%) & $\begin{array}{l}\text { Mittelwert } \bar{x} \text { mit Standardab- } \\
\text { weichung } \\
\text { (SD) }\end{array}$ \\
\hline \multicolumn{2}{|l|}{ Geschlecht } & \multirow[t]{3}{*}{-} \\
\hline Männlich & $5(41,7)$ & \\
\hline Weiblich & $7(58,3)$ & \\
\hline \multicolumn{2}{|l|}{ Alter } & \multirow[t]{6}{*}{$46( \pm 12,22)^{a}$} \\
\hline 26-35 Jahre & $3(25,0)$ & \\
\hline 36-45 Jahre & $1(8,3)$ & \\
\hline 46-55 Jahre & $4(33,3)$ & \\
\hline 56-65 Jahre & $3(25,0)$ & \\
\hline Keine Angabe & $1(8,3)$ & \\
\hline \multicolumn{2}{|l|}{ Berufserfahrung } & \multirow[t]{7}{*}{$20,77( \pm 13,42)^{a}$} \\
\hline 0-10 Jahre & $4(33,3)$ & \\
\hline 11-20 Jahre & $1(8,3)$ & \\
\hline 21-30 Jahre & $3(25,0)$ & \\
\hline 31-40 Jahre & $2(16,7)$ & \\
\hline 41-50 Jahre & $1(8,3)$ & \\
\hline Keine Angabe & $1(8,3)$ & \\
\hline \multicolumn{2}{|l|}{ Setting } & \multirow[t]{3}{*}{-} \\
\hline Land & $6(50,0)$ & \\
\hline Kommune & $6(50,0)$ & \\
\hline \multicolumn{2}{|c|}{$\begin{array}{l}\text { Stadt- und Gemeindetyp (nach Bundesamt für Bauwesen und } \\
\text { Raumordnung) }\end{array}$} & \multirow[t]{5}{*}{-} \\
\hline Großstadt & $3(50,0)$ & \\
\hline Mittelstadt & $2(33,3)$ & \\
\hline Kleinstadt & $1(16,7)$ & \\
\hline Landgemeinde & 0 & \\
\hline \multicolumn{2}{|c|}{$\begin{array}{l}\text { Bildungsniveau (nach International Standard Classification of } \\
\text { Education (ISCED)) }\end{array}$} & \multirow[t]{5}{*}{-} \\
\hline ISCED 4 & $1(8,3)$ & \\
\hline ISCED 6 & $2(16,7)$ & \\
\hline ISCED 7 & $7(58,3)$ & \\
\hline ISCED 8 & $2(16,7)$ & \\
\hline \multicolumn{2}{|l|}{ Ausbildungsfeld } & \multirow[t]{7}{*}{-} \\
\hline $\begin{array}{l}\text { Sport-, Gesundheitswissen- } \\
\text { schaften }\end{array}$ & $5(41,7)$ & \\
\hline Verwaltungswissenschaften & $2(16,7)$ & \\
\hline Ingenieurswissenschaften & $1(8,3)$ & \\
\hline Sozialwissenschaften & $2(16,7)$ & \\
\hline Naturwissenschaften & $1(8,3)$ & \\
\hline Sprach-, Kulturwissenschaften & $1(8,3)$ & \\
\hline \multicolumn{2}{|l|}{ Berufstätigkeit } & \multirow[t]{5}{*}{-} \\
\hline Bereichsleitung & $4(33,3)$ & \\
\hline Referentin/Referent & $4(33,3)$ & \\
\hline Sachbearbeitung & $2(16,7)$ & \\
\hline Sonstiges & $2(16,7)$ & \\
\hline \multicolumn{3}{|l|}{ afehlende Angabe $(n=1)$} \\
\hline
\end{tabular}

der Fälle und Kontexte innerhalb des sozioökonomischen Deprivationsindexes für Deutschland des Robert KochInstituts auf Bundeslandebene aus dem Jahr 2014 [21] ebenfalls gezielt ausgewählt (Purposive Sampling). • Abb. 1 fasst das Vorgehen zusammen. Die Kontaktaufnahme bei 85 Personen erfolgte telefonisch oder per E-Mail (Rückmeldung von 35 Personen), dabei wurden Ziele und Hintergründe der Interviews vorgestellt und datenschutzkonforme Einwilligungserklärungen per E-Mail versendet. Bei schriftlicher Einwilligung zur Teilnahme wurde ein Interviewtermin vereinbart. Als Gründe für die Nichtteilnahme wurden u. a. fehlende zeitliche Ressourcen und Kompetenzen von den $\mathrm{AK}$ angegeben.

Insgesamt wurden zwischen Juni 2019 und August 202010 telefonische und persönliche Interviews im Arbeitsumfeld von 12 Expertinnen und Experten bis zur thematischen Sättigung von der Erstautorin durchgeführt. Bei den Interviews handelt es sich zumeist um Einzelinterviews, lediglich ein Interview wurde als Gruppeninterview geführt. Die Dauer betrug zwischen $15 \mathrm{~min}$ und $54 \mathrm{~min}$. Die Interviews wurden tontechnisch aufgezeichnet, transkribiert und mittels qualitativer Inhaltsanalyse nach Gläser und Laudel [22] im Vieraugenprinzip mithilfe von Microsoft Excel (Microsoft Corporation, Redmond, WA, USA) codiert und ausgewertet (siehe Onlinematerial Tabelle Z1). Die qualitative Inhaltsanalyse enthielt dabei 5 zentrale Schritte: theoretische Vorüberlegungen, Vorbereitung der Extraktion, Extraktion, Aufbereitung und Auswertung (siehe Beschreibung der Datenanalyse im Onlinematerial).

\section{Ergebnisse}

Im Folgenden wird ein Teil der zentralen Ergebnisse der Studie anhand der Kategorien Informationsbeschaffung und WE (- Tab. 1) und den im Onlinematerial Tabelle Z1 genannten Dimensionen vorgestellt. - Tab. 1 und Tabelle Z1 fassen die verschiedenen Kategorien und Subkategorien mit beispielhaften Zitaten zusammen. Die soziodemografischen Merkmale der Teilnehmenden können - Tab. 2 entnommen werden. 


\section{Kategorie Informationsbeschaffung}

Um Bewegungsförderungsmaßnahmen in der Kommune etablieren oder weiterentwickeln zu können, müssen kommunale AK Informationen und Daten beschaffen und nutzen. Dabei kommen laut der interviewten AK 5 Zugangswege infrage:

- Personen im direkten Umfeld (z.B. Kollegen),

- Netzwerke (z. B. Städte- und Gemeindebünde),

- Internet,

- Newsletter und

- Fachzeitschriften.

Die Zugangswege und Rahmenbedingungen sind dabei stark von den jeweiligen AK, ihren Vorkenntnissen, Erfahrungen und Tätigkeitsbereichen abhängig und folglich aufgrund der $A k$ teursvielfalt im Bereich der Bewegungsförderung heterogen. Problematisch ist die fehlende Zeit zum Lesen und Verstehen von Informationen und Daten. So werden Materialien durch einen Mangel an Ressourcen häufig nicht gelesen. Die $\mathrm{AK}$ bemängeln die Masse an Input, die $\mathrm{zu}$ einem Informationsüberfluss führen kann.

2. Kategorie Wissenschaftliche Erkenntnisse

Die interviewten AK sehen einen Nutzen in WE und verstehen sie insbesondere als Umsetzungs- und Argumentationshil$f e$ gegenüber Politik und Einwohnenden. Die WE werden in Entscheidungen einbezogen, die Relevanz schwankt je nach AK und Aufgabenspektrum. Um politischen Rückhalt zu erhalten, ist eine wissenschaftliche Fundierung zumeist unumgänglich, jedoch sind die vorhandene Komplexität und ein zu großer Umfang in Kombination mit knappen Ressourcen oftmals abschreckende Barrieren. Statistik und Fachsprache sind zum Teil schwer verständlich und WE für die AK schwer zugänglich. Lediglich einer der befragten AK verneint das Vorhandensein von Barrieren.

ZurBeseitigung dieser Barrieren wünschen sich die AK im Sinne der strukturellen Aufbereitung Suchmasken und Filter zur Durchsicht der Literatur so- wie kurze Zusammenfassungen in allgemeinverständlicher Sprache. In Bezug auf die Länge sind sich die AK uneinig, sodass eine Spanne zwischen einer und 30 Seiten genannt wird. Die Mehrheit plädiert jedoch für ein möglichst kurzes Format. Die AK merken zur inhaltlichen Aufbereitung an, dass WE Informationen zur Übertragbarkeit in den eigenen Kontext, zu förderlichen und hinderlichen Faktoren, Setting und Rahmenbedingungen enthalten sollten. Sie sind sich einig, dass Angaben zu Kosten (z. B. Kostenarten) für eine bessere Maßnahmenplanung gemacht werden müssten. Eine Aufführung von Beispielen sehen alle AK als hilfreich an, währenddessen in Bezug auf die Auflistung von statistischen Werten Uneinigkeit besteht. Das Ergebnis der WE sollte abschließend kurz und prägnant mit Handlungsempfehlungen dargestellt werden.

Ein Großteil der AK bevorzugt die Bereitstellung von WE per Newsletter, da nach Inhalten selektiert werden kann und nicht aktiv nach Literatur gesucht werden muss. Lediglich ein Akteur präferiert eine Bereitstellung per Fachzeitschrift. Weiterhin sind Transferworkshops auf Veranstaltungen und telefonische sowie persönliche Beratungen vorstellbar, sodass Fragen direkt beantwortet und Probleme behoben werden können.

\section{Diskussion}

Diese qualitative Studie gibt erstmalig im Bereich der kommunalen Bewegungsförderung in Deutschland Hinweise auf die Komplexität der Zugangswege zu und Aufbereitung von WE. Die Interviewten sehen den Nutzen der Wissenschaft; jedoch bestehen vielseitige Barrieren, die auch in nationalen und internationalen Forschungsarbeiten über verschiedene Zielgruppen hinweg bestätigt wurden [2, $5,6,23-29]$. Sowohl in dieser als auch in weiteren Untersuchungen konnte festgestellt werden, dass Zusammenfassungen mit entscheidungsrelevanten Angaben in allgemeinverständlicher Sprache signifikant für die Praxis sind und zu einer häufigeren Nutzung führen $[2,4-6,13$, $17,23,24,30,31]$. Die scheinbar widersprüchliche Vorliebe für Kürze und gleichzeitig Detailliertheit wurde auch von anderen Autorinnen und Autoren [6, 31] berichtet. Weitere Studien bestärken zum Teil die von den AK bevorzugten Kanäle für den Erhalt von WE [4, 6, 13, 29]. Diese und andere [4, 9, 29, 32] zeigen jedoch auch, dass Onlinezugänge, Webseiten und soziale Medien wirksamere Strategien für die Verbreitung von WE sind. Die bevorzugten Zugangswege sollten daher spezifisch bei verschiedenen Zielgruppen und Themen erneut abgefragt werden.

Partizipative Wissenstranslation wurde im Rahmen dieser Studie nicht untersucht. In der Literatur gelten als Erfolgsfaktoren für die Nutzung und Akzeptanz von WE ein rechtzeitiger Zugang zu qualitativ hochwertigen und relevanten Forschungsergebnissen, die Zusammenarbeit von Forschenden mit Entscheidungstragenden und der Aufbau von Partnerschaften zwischen Forschenden und praktisch Anwendenden [23, 26, 33-36]. Eine mögliche Schlussfolgerung daraus wäre, Wissenstranslation als zyklisch und nicht unidirektional anzusehen. Dies bedeutet, Wissen nicht nur aus der Forschung in die Praxis $\mathrm{zu}$ bringen und von Beginn der Forschungsbemühungen zu berücksichtigen, sondern auch aus der Praxis in die Forschung zu geben [37], damit Bedarfe mitgeteilt und Erfahrungen einfließen können. Insbesondere partizipatorische, interaktive Ansätze, die die $\mathrm{AK}$ aktiv in Forschungsprojekte einbeziehen, beispielsweise in Form von What-WorksPapieren aus Kanada [38] oder den Niederlanden [39], haben sich im internationalen Raum im Vergleich zu unidirektionalen Modellen als vielversprechend herausgestellt $[9,40]$. Hierbei sollten mögliche Limitationen wie die Kompetenzen, Vorerfahrungen und zeitlichen Ressourcen der AK beachtet und untersucht werden.

Bundesaufgabe kann in einem ersten Schritt daher die Vernetzung von AK der Bewegungsförderung aus Praxis, Politik und Wissenschaft durch bspw. niedrigschwellige Veranstaltungen sein. Persönliche Begegnungen haben sich in der Vergangenheit dabei als effizientester Weg herausgestellt $[34,36]$. Des Weiteren bietet sich die Bereitstellung einer einheitlichen, leicht zugänglichen 
und in Partizipation mit den AK erarbeiteten digitalen Plattform an, die ein standardisiertes Vorgehen der kommunalen Bewegungsförderung trotz Varianz in den Strukturen zulässt, für die Praxis relevante, aktuelle und bedarfsgerecht aufbereitete WE bündelt, ggfs. per Newsletter zur Verfügung stellt und so die „Pull“-Bemühungen der AK fördert. Die Plattform kann zugleich für Feedback und die Entwicklung von praxisbasierten, wissenschaftlichen Fragestellungen in einem bedürfnisgerechten Format (ähnlich What Works) genutzt werden. Eine derartige Plattform (Impulsgeber Bewegungsförderung) wird derzeit u. a. aufbauend auf diesen Ergebnissen im Rahmen einer entsprechenden Implementierungsstrategie von der $\mathrm{BZgA}$ entwickelt. Im internationalen Raum haben sich ähnliche Plattformen als hilfreiche Instrumente für unterschiedliche Nutzende herausgestellt [36, 41, 42], die Untersuchung der Effektivität erweist sich jedoch als Herausforderung [36]. Um die Durchdringung der Maßnahmen zu erhöhen, könnte dieses Angebot durch analoge Beratungsleistungen ergänzt werden. Hier könnten beispielsweise, ähnlich zum Konzept des Knowledge Broker [43], Personen oder Organisationen als Wissensvermittler eingesetzt werden. Alternativ ist auch das Angebot von Kompetenzschulungen und Trainings für praktisch Anwendende und Forschende vorstellbar. Integrierte Interventionen aus Kompetenzentwicklung und dem Zugang zu Wissensvermittlern, Ressourcen und Tools haben sich dabei in bisherigen Forschungsarbeiten als vielversprechende Strategie herausgestellt [35].

\section{Limitationen}

Diese Studie spiegelt die vielfältigen Bedürfnisse von interdisziplinär ausgebildeten $\mathrm{AK}$ der Bewegungsförderung auf Kommunal- und Landesebene wider. Diese sind jedoch möglicherweise nicht auf andere Themenbereiche außerhalb der kommunalen Bewegungsförderung übertragbar und für AK von Kommunen mit geringen Einwohnendenzahlen (Landgemeinden, - Tab. 2) anwendbar. Die AK waren sich bewusst, dass die
Interviewerin für eine Bundesbehörde arbeitet, was das Antwortverhalten beeinflusst haben und zu Verzerrungen geführt haben könnte.

\section{Fazit}

Zum ersten Mal wurden in einer qualitativen Studie AK der Bewegungsförderung von älteren Menschen in Deutschland zu den Zugangswegen, der Aufbereitung und Bereitstellung von WE interviewt. Die Translation von WE ist für AK zwar äußerst relevant, jedoch mangelt es an praxisgerechten Aufbereitungen und Zugängen. Damit künftig WE häufiger in der Praxis Anwendung finden und evidenzinformierte Entscheidungen getroffen werden, sollten WE bedarfs- und bedürfnisgerecht aufbereitet und verbreitet werden.

\section{Korrespondenzadresse}

\section{Annalena Bußkamp}

Referat 2-22 „Zusammenarbeit mit Ländern,

Krankenkassen und Verbänden, Gremien;

Gesundes Alter; Frauengesundheit;

Männergesundheit", Bundeszentrale für gesundheitliche Aufklärung

Maarweg 149-161, 50825 Köln, Deutschland

annalena.busskamp@bzga.de

Danksagung. Unser besonderer Dank gilt allen interviewten Expertinnen und Experten für den interessanten Input sowie David Litaker und Anne Bruns für die methodische und inhaltliche Beratung.

\section{Einhaltung ethischer Richtlinien}

Interessenkonflikt. A. Bußkamp, C. Vonstein, J. Tillmann, R. Roßmann und F. De Bock geben an, dass kein Interessenskonflikt besteht. Die inhaltliche Durchführung des Projekts und der Studie erfolgt unabhängig von der finanziellen Unterstützung durch den Verband der Privaten Krankenversicherung e. V.

Für diesen Beitrag wurden von den Autoren keine Studien an Menschen oder Tieren durchgeführt. Für die aufgeführten Studien gelten die jeweils dort angegebenen ethischen Richtlinien.

Die Studie zur Qualitätssicherung wurde mit dem für Datenschutz zuständigen Justiziar der BZgA abgestimmt und auf Basis dessen Einverständniserklärungen nach Artikel 7 der Datenschutzgrundverordnung umgesetzt. Von allen beteiligten Interviewten liegt eine schriftliche Einverständniserklärung, auch für die Publikation, vor. Die Notwendigkeit der Einholung eines offiziellen Ethikvotums einer medizinischen Ethikkommission bestand gemäß der Guideline der Deutschen Forschungsgemeinschaft für die Sozialwissenschaften nicht [44], da an der Studie keine Pa- tientinnen, Patienten oder Personen mit besonderem Schutzbedürfnis beteiligt waren, keine personenbezogenen gesundheitlichen Daten erhoben wurden und die Betroffenen keinen besonderen Risiken außerhalb ihrer alltäglichen Erfahrungen ausgesetzt waren.

Open Access. Dieser Artikel wird unter der Creative Commons Namensnennung 4.0 International Lizenz veröffentlicht, welche die Nutzung, Vervielfältigung, Bearbeitung, Verbreitung und Wiedergabe in jeglichem Medium und Format erlaubt, sofern Sie den/die ursprünglichen Autor(en) und die Quelle ordnungsgemäß nennen, einen Link zur Creative Commons Lizenz beifügen und angeben, ob Änderungen vorgenommen wurden.

Die in diesem Artikel enthaltenen Bilder und sonstiges Drittmaterial unterliegen ebenfalls der genannten Creative Commons Lizenz, sofern sich aus der Abbildungslegende nichts anderes ergibt. Sofern das betreffende Material nicht unter der genannten Creative Commons Lizenz steht und die betreffende Handlung nicht nach gesetzlichen Vorschriften erlaubt ist, ist für die oben aufgeführten Weiterverwendungen des $\mathrm{Ma}$ terials die Einwilligung des jeweiligen Rechteinhabers einzuholen.

Weitere Details zur Lizenz entnehmen Sie bitte der Lizenzinformation auf http://creativecommons.org/ licenses/by/4.0/deed.de.

\section{Literatur}

1. National Collaborating Centre for Methods and Tools (2021) Evidence-informed public health. https://www.nccmt.ca/tools/eiph. Zugegriffen: 4. Jan. 2021

2. Lavis J, Davies H, Oxman A, Denis JL, GoldenBiddle K, Ferlie E (2005) Towards systematic reviews that inform health care management and policy-making. J Health Serv Res Policy 10(1):35-48

3. Straus SE, Tetroe JM, Graham ID (2011) Knowledge translation is the use of knowledge in health care decision making. JClin Epidemiol 64:6-10

4. Dobbins M, Rosenbaum P, Plews N, Law M, Fysh A (2007) Information transfer: what do decision makers want and need from researchers? Implement Sci 2:20

5. Brownson RC, Fielding JE, Green LW (2018) Building capacity for evidence-based public health: reconciling the pulls of practice and the push of research. Annu Rev Public Health 39:27-53

6. Busert LK, Mütsch M, Kien Cet al (2018) Facilitating evidence uptake: development and user testing of a systematic review summary format to inform public health decision-making in Germanspeaking countries. Health Res Policy Syst 16:59

7. Lavis JN (2009) How can we support the use of systematic reviews in policymaking? PLoS Med 6:e1000141

8. Böhme C, Reimann B (2018) Integrierte Strategien kommunaler Gesundheitsförderung. Rahmenbedingungen, Steuerung und Kooperation. Ergebnisse einer Akteursbefragung. Deutsches Institut für Urbanistik-Difu-, Berlin

9. Brownson RC, Fielding JE, Maylahn CM (2009) Evidence-based public health: a fundamental concept for public health practice. Annu Rev Public Health 30:175-201

10. Jewell C, Bero L (2008) "Developing good taste in evidence": facilitators of and hindrances to 
evidence-informed health policymaking in state government. Milbank Quarterly 86:177-208

11. South E, Lorenc T (2020) Use and value of systematic reviews in English local authority public health: a qualitative study. BMC Public Health 20:1100

12. Kelly MP, Atkins L, Littleford C, Leng G, Michie S (2017) Evidence-based medicine meets democracy: the role of evidence-based public health guidelines in local government. J Public Health (Oxf) 39:678-684

13. Haas S, Breyer E, Knaller C, Weigl M (2013) Evidenzrecherche in der Gesundheitsförderung (Wissen 10 Teil 1 Handbuch)

14. Khoddam H, Mehrdad N, Peyrovi H, Kitson AL, Schultz TJ, Athlin AM (2014) Knowledge translation in health care: a concept analysis. Med J Islam Republran 28:98-98

15. Canadian Institutes of Health Research (2019) Knowledge translation: definition. https://cihrirsc.gc.ca/e/29529.html.. Zugegriffen: 04. Jan. 2021

16. Graham ID, Logan J, Harrison MB et al (2006) Lost in knowledge translation: time for a map? J Contin Educ Health Prof 26:13-24

17. Ellen ME, Panisset U, Araujo de Carvalho I, Goodwin J, Beard J (2017) A knowledge translation framework on ageing and health. Health Policy 121:282-291

18. Tong A, Sainsbury P, Craig J (2007) Consolidated criteria for reporting qualitative research (COREQ): a 32-item checklist for interviews and focus groups. Int JQual Health Care 19:349-357

19. Kruse J (2015) Qualitative Interviewforschung. Ein integrativer Ansatz. Beltz Juventa, Weinheim, Basel

20. Bumiller M, Hübler M, Simen J (2015) Wissensmanagement in der öffentlichen Verwaltung

21. Kroll LE, Schumann M, Hoebel J, Lampert T (2017) Regionale Unterschiede in der Gesundheit - Entwicklung eines sozioökonomischen Deprivationsindex für Deutschland. J Health Monit 2:103-120

22. Gläser J, Laudel G (2010) Experteninterviews und qualitative Inhaltsanalyse. SpringerVS, Wiesbaden

23. Tricco AC, Cardoso R, Thomas SM et al (2016) Barriers and facilitators to uptake of systematic reviews by policy makers and health care managers: a scoping review. Implement Sci 11:4

24. Lavis J, Catallo C, Permanand G, Zierler A, BRIDGE Study Team (2013) Communicating clearly: enhancing information-packaging mechanisms to support knowledge brokering in European health systems

25. Allen $P$, Jacob RR, Lakshman M, Best LA, Bass K Brownson RC (2018) Lessons learned in promoting evidence-based public health: perspectives from managers in state public health departments. JCommunity Health 43:856-863

26. Oliver K, Innvar S, Lorenc T, Woodman J, Thomas J (2014) A systematic review of barriers to and facilitators of the use of evidence by policymakers. BMC Health Serv Res 14:2

27. Harris JK, Beatty K, Leider JP, Knudson A Anderson BL, Meit M (2016) The double disparity facing rural local health departments. Annu Rev Public Health 37:167-184

28. Petkovic J, Welch V, Jacob MH et al (2018) Do evidence summaries increase health policymakers' use of evidence from systematic reviews? A systematic review. Syst Rev 14:1-52

29. Brownson RC, Eyler AA, Harris JK, Moore JB, Tabak RG (2018) Getting the word out: new approaches for disseminating public health science. J Public Health Manag Pract 24:102-111
30. Ellen M, Lavis J, Wilson Met al (2014) Health system decision makers' feedback on summaries and tools supporting the use of systematic reviews: a qualitative study. Evid Policy. https://doi.org/10. 1332/174426514X672362

31. Rosenbaum SE, Glenton C, Wiysonge CSetal (2011) Evidence summaries tailored to health policymakers in low- and middle-income countries. Bul World Health Organ 89:54-61

32. Perrier L, Mrklas K, Lavis JN, Straus SE (2011) Interventions encouraging the use of systematic reviews by health policymakers and managers: a systematic review. Implement $\mathrm{Sci}$ 6:43

33. Innvaer S, Vist G, Trommald M, Oxman A (2002) Health policy-makers' perceptions of their use of evidence: a systematic review. J Health Serv Res Policy 7:239-244

34. Jansen MW, van Oers HA, Kok G, de Vries NK (2010) Public health: disconnections between policy, practice and research. Health Res Policy Syst 8:37-37

35. Armstrong R, Waters E, Dobbins M et al (2013) Knowledge translation strategies to improve the use of evidence in public health decision making in local government: intervention design and implementation plan. Implement Sci 8:121

36. Pettman TL, Armstrong R, Johnson S et al (2020) Evaluation outcomes of a knowledge translation platform: a structure for support and exchange in prevention. Evid Policy 16:99-121

37. Vollmar HC, Santos S, de Jong A, Meyer G, Wilm S (2017) Wie gelangt Wissen in die Versorgung? Bundesgesundheitsblatt Gesundheitsforschung Gesundheitsschutz 60:1139-1146

38. Bornstein S, Baker R, Navarro P, Mackey S, Speed D Sullivan M (2017) Putting research in place: an innovative approach to providing contextualized evidence synthesis for decision makers. Syst Rev 6:218-218

39. NijlandS, PrellerL, Kalkmanen I, Willemsen N(2018) Werkzame elementen van beweeginterventies voor 55-plussers

40. Rütten A, Wolff A, Streber A (2016) Interaktiver Wissenstransfer in der Gesundheitsförderung: das GESTALT-Projekt. Erste Ergebnisse der Erprobung eines Ansatzes zur nachhaltigen Implementierung evidenzbasierter Bewegungsprogramme. Gesundheitswesen 78:359-366

41. Pettman TL, Armstrong R, Waters E et al (2016) Evaluation of a knowledge translation and exchangeplatformtoadvancenon-communicable disease prevention. Evid Policy 12:109-126

42. Yamada J, Shorkey A, Barwick M, Widger K, Stevens BJ (2015) The effectiveness of toolkits as knowledge translation strategies for integrating evidence into clinical care: a systematic review. BMJOpen 5:e6808

43. Ward V, House A, Hamer S (2009) Knowledge brokering: the missing link in the evidence to action chain? Evid Policy 5:267-279

44. Deutsche Forschungsgemeinschaft (2020) FAQ: informationen aus den Geistes- und Sozialwissenschafte. https://www.dfg.de/foerderung/faq/ geistes_sozialwissenschaften/.Zugegriffen:4.Jan. 2021 